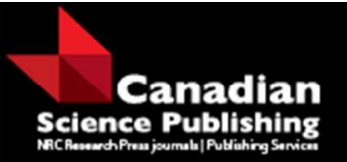

Canadian Journal of Forest Research Revue canadienne de recherche forestière

\title{
Forest damage inventory using the local pivotal sampling method
}

\begin{tabular}{|r|l|}
\hline Journal: & Canadian Journal of Forest Research \\
\hline Manuscript ID & cjfr-2016-0411.R1 \\
\hline Danuscript Type: & Article \\
\hline Complete List of Authors: & $\begin{array}{l}\text { Roberge, Cornelia; Sveriges Lantbruksuniversitet Fakulteten for } \\
\text { Skogsvetenskap, Forest resource management } \\
\text { Grafström, Anton; Swedish University of Agricultural Sciences, Department } \\
\text { of Forest Resource Management } \\
\text { Stăhl, Göran; Swedish University of Agricultural Sciences, Forest Resource } \\
\text { Management }\end{array}$ \\
\hline Keyword: & $\begin{array}{l}\text { Forest damage inventory, Forest health, Local pivotal method, Two-phase } \\
\text { sampling for stratification, Double sampling }\end{array}$ \\
\hline
\end{tabular}

\section{SCHOLARONE ${ }^{m}$}

Manuscripts 


\section{Forest damage inventory using the local pivotal sampling method}

2 Cornelia Roberge, Anton Grafström and Göran Ståhl

3 Cornelia Roberge

4 Department of Forest Resource Management,

5 Swedish University of Agricultural Sciences (SLU),

690183 Umeå, Sweden.

$7 \quad$ Fax: $+46(0) 90778116$

$8 \quad$ Tel: $+46(0) 907868370$

9 e-mail: cornelia.roberge@slu.se

10 Anton Grafström

11 Department of Forest Resource Management,

12 Swedish University of Agricultural Sciences (SLU),

1390183 Umeå, Sweden.

14 e-mail: anton.grafstrom@slu.se

15 Göran Ståhl

16 Department of Forest Resource Management,

17 Swedish University of Agricultural Sciences (SLU),

1890183 Umeå, Sweden.

19 e-mail: goran.stahl@slu.se 


\section{Abstract}

22 Specially designed forest damage inventories, directed to areas with potential or suspected damage, are

23 performed in many countries. In this study we evaluate a new approach for damage inventories where

24 auxiliary data are used for the sample selection with the recently introduced local pivotal sampling

25 design. With this design, a sample that is well spread in the space of the auxiliary variables is obtained.

26 We applied Monte Carlo sampling simulation to evaluate whether this sampling design leads to more

27 precise estimates compared to commonly applied baseline methods. The evaluations were performed

28 using different damage scenarios and different simulated relationships between the auxiliary data and

29 the actual damages. The local pivotal method was found to be more efficient than simple random

30 sampling in all scenarios and, depending on the allocation of the sample and the properties of the

31 auxiliary data, it sometimes outperformed two-phase sampling for stratification. Thus, the local pivotal

32 method may be a valuable tool to cost-efficiently assess the magnitude of forest damage once

33 outbreaks have been detected in a forest region.

Keywords: Forest damage inventory, Forest health, Local pivotal method, Two-phase sampling for 36 stratification, double sampling

Résumé

Dans plusieurs pays, des inventaires de dommages forestiers spécialement conçus sont utilisés dans des régions avec dommages potentiels ou soupçonnés. Dans la présente étude, nous évaluons une nouvelle

42 approche pour les inventaires de dommages forestiers où des données auxiliaires sont utilisées pour la

43 sélection de l'échantillon en utilisant la méthode du pivot local d'échantillonnage. Cette approche

44 permet d'obtenir un échantillon qui est bien réparti dans l'espace des variables auxiliaires. Nous avons 
45

46

47

\section{Introduction}

utilisé des simulations Monte Carlo pour évaluer si cette méthode donne des estimés plus précis comparativement à d'autres méthodes communes. Les évaluations ont été faites en utilisant différents scénarios de dommage et différentes relations simulées entre les données auxiliaires et le dommage réel. La méthode du pivot local s'est avéré plus efficace que l'échantillonnage aléatoire simple pour tous les scénarios et, dépendant de la répartition de l'échantillon et des propriétés des données auxiliaires, elle surpassait parfois l'échantillonnage à deux phases pour stratification. En somme, la méthode du pivot local peut être un outil de choix pour l'évaluation efficace de l'amplitude des dommages forestiers lorsque des épidémies ont été détectées dans une région donnée.

The world's forests provide numerous ecosystem services (e.g. Mery et al. 2005). However, concerns

56 have been raised that the world's forests are under increasing stress due to global change (Trumbore, et

57 al. 2015). One example is stress to forest trees due to heat and drought (Allen, et al. 2010) which may 58 increase forest damage (Dale et al. 2001), thereby potentially limiting the forests' capacity to provide 59 ecosystem services (Mery et al. 2005). As part of responsible forest management, many countries have developed forest health monitoring schemes for identifying and investigating potential threats to the

61 forest resource, e.g. USA (Bennett and Tkaz 2008), Australia (Carnegie 2008), Sweden (Wulff et al. 2012)

62 and Norway (Aamlid et al. 2000). In many cases the schemes include some form of aerial detection 63 survey or sketch-mapping (e.g. Backsen and Howell, 2013), and the usefulness of remotely sensed data 64 is increasingly acknowledged (Hall et al. 2016). In addition to detecting the presence of damage, there is 65 often a need to identify the damaging agent and to accurately quantify the extent of damage for informed decisions (Wulff et al., 2012). This usually requires data obtained from field inventories,

67 whether it is used for training or validating a model to map the extent of damage, or for producing a 68 statistical estimate (e.g. the number of damage trees, or affected volume/area). It is important to have 
reliable information for which the uncertainty can be specified. Therefore, estimates based on designbased inference are often required (Ferretti, 2009). In a study by Roberge et al (2016) it was found that great gains in precision can be obtained with two-stage-sampling for stratification, using existing national forest inventory plots as a first phase. Moreover, using forest damage maps for poststratification improved the precision further.

There is ongoing work in the field of mathematical statistics where new sampling methods that utilize auxiliary variables for selecting samples are being developed. A sample that is well spread, i.e. balanced in the auxiliary space, provides a good representation of the sampled population (Grafström and Schelin 2013, Grafström and Lundström 2013) and thus results in precise estimates of the target parameter in case there is a strong relationship between the target variable and the auxiliary variables. The local pivotal method (LPM) was introduced by Grafström et al. (2012). The LPM utilizes distance in the auxiliary space to identify similar units that compete for inclusion in the sample. In that way the LPM avoids selection of nearby units and forces the sample to become well spread. It has recently been evaluated in the estimation of forest variables such as basal area, standing volume and mean height (Grafström and Ringvall, 2013, Grafström et al. 2014). The LPM method of sample selection is now available in the R (R Core Team 2016) package BalancedSampling (Grafström and Lisic, 2016).

The aim of this study was to provide a first test of LPM in the context of forest damage inventory. For comparison, two well-known baseline methods were also evaluated: simple random sampling (SI) and two-phase sampling for stratification (2PS) with a systematic random sample (SYS) in the first phase and stratified random sampling (STSI) in the second phase. Here, two strata were used for the STSI: grid-cells classified as damaged or undamaged. With the LPM method, the samples were spread geographically and in the space of auxiliary data. The efficiencies of the three different sampling strategies were compared through Monte Carlo simulation, using simulated forest damage populations of spruce bark 
93 beetle (Ips typographus) and simulated auxiliary data in the form of damage tree counts, mimicking

94 auxiliary data that could be obtained from, e.g., interpretation of aerial photos or a systematic helicopter inventory of each grid-cell in an area of interest. Remotely sensed data for directing the sample selection might also be obtained from other sources; such as digital color-infrared aerial

97 photographs (Murtha and Wiart 2010), high resolution satellite imagery (Hais et al. 2009), satellite radar

98 (Ortiz et al. 2013), airborne laser scanner data (Solberg 2010) or hyperspectral imaging (Näsi et al. 2015).

99 For good back-ground and reviews of remote sensing of forest damage we refer to Hall et al. (2016),

100 Rullan-Silva et al. (2013) and Ciesla (2000).

101

102

\section{Materials and Methods}

The different sampling strategies were applied for estimating the number of damage trees in four different simulated populations of bark beetle damage. We used a quadrat-shaped area of interest (AOI) located in the Swedish county of Västernorrland (cf. Roberge et al. 2016) to represent a typical municipality of $900 \mathrm{~km}^{2}$, divided into $1,200 \times 1,200$ grid-cells of $25 \times 25$ meters each. The AOI had a forest cover of $80 \%$ and comprised 13,870 forest stands in the segmented kNN-Sweden data (Reese et al. 2003) used for simulating the damage populations (Figure 1, Table 1). The auxiliary data used for the sample selection were the spatial coordinates $(X Y)$ of the grid-cells, damage tree counts (DTC, i.e. numbers of detected damage trees in grid-cells), and grid-cells classified as containing damage trees (D) or not (a binary variable). The 2PS and LPM designs start with a first-phase large systematic sample for which XY, DTC, and D auxiliary data were made available (the first phase sample sizes are 10,000 and 3,600). Second phase samples were drawn (with sample sizes 50, 100, 150 and 200), using either LPM or STSI, from this systematic sample.

SI does not use any of the auxiliary information and the sample is selected entirely at random from the population of grid-cells. For the 2PS design we used the D auxiliary data for partitioning the first-phase 
116 sample selected from the AOI into two strata. In each stratum, SI sampling was applied. LPM was used

117 with equal probabilities, including grid-cells where no damage was detected (DTC=0).

\section{Inventory strategies}

119 The true number of damage trees in the AOI is the sum of damage trees over all grid-cells in the area:

$$
Y=\sum_{i=1}^{N} y_{i}
$$

where $N$ is the total number of grid-cells in the AOI and $y_{i}$ is the number of damage trees in grid-cell $i$.

122 The baseline inventory methods are two commonly used sampling designs: SI from the whole AOI, and

$1232 \mathrm{PS}$, which utilizes the auxiliary information in sample selection. The two baseline methods were

124 compared with LPM, which uses multiple auxiliary data sources for the sample selection. 2PS has been

125 frequently applied in forest damage inventories over the years (Lucas 2009, Roberge et al. 2016). Both

126 LPM and 2PS were based on an initial systematic sample of $n_{1}$ grid-cells spread in geographical (XY)

127 space. For the grid-cells sampled in the first phase, auxiliary data (XY, DTC and D) were made available.

128 From the first-phase sample a second-phase sample was selected using LPM or STSI designs. The LPM

129 spread the second phase sample in the XY and DTC auxiliary data spaces with equal inclusion

130 probabilities (in this application). The 2PS design used the D auxiliary space to apply STSI sampling in the

131 second phase, with predetermined sample sizes. In STSI, 20 or $40 \%$ of the sample was allocated to the

132 strata without detected damage and the rest of the sample to the stratum where $D T C \geq 1$ (i.e. $D=1$ ). The

133 two different allocation principles were selected in order to assess whether a larger or a smaller

134 proportion of the sample should be selected in the stratum without detected damage, although in both

135 cases the majority of the plots were allocated to the stratum with detected damage. This led to two sets

136 of results for the 2PS design denoted '2PS_20' (20\%) and '2PS_40' (40\%). For both 2PS and LPM we

137 investigated the impacts of different errors in the auxiliary data, in the form of errors of omission and

138 commission of different sizes. 
139

140

141

142

143

144

145

146

147

148

149

150

151

\section{Estimators}

We used the $\pi^{*}$-estimator (Särndal et al. 1992, p. 348) where each included grid-cell value is divided by its inclusion probability. This results in the regular HT-estimator for the population total, for LPM:

where $N$ is all grid-cells, $n_{1}$ is the first-phase sample size and $n$ is the sample size selected in the second phase. The mean, $\overline{y_{S}}$ is the sample mean from the second phase (S2) sample. For the 2PS design (Särndal et al. 1992, p. 353) we used the following estimator for the population total

$$
\widehat{Y}_{2 P S}=N \sum_{h=1}^{H_{S 1}} w_{a h} \bar{y}_{S h}=N \hat{\bar{y}}_{U}
$$

where $w_{a h}=n_{1, h} / n_{1}$ (i.e. the relative size of stratum $h$ in the first phase sample), and $\bar{y}_{s h}$ the mean of the value of interest within stratum $h$. In this application only two strata were used $\left(H_{S 1}=2\right)$. The estimated mean over all grid-cells $(U)$ in the AOI is denoted $\hat{\bar{y}}_{U}$.

For the SI design the following estimator for the population total was used

$$
\hat{Y}_{S I}=\frac{N}{n} \sum_{i=1}^{n} y_{i}=N \bar{y}_{S}
$$

\section{2}

\section{Variance estimation}

153 The variance of samples that are well-spread in auxiliary space can be estimated by applying the

154 following estimator:

155

$$
\widehat{V}_{G S}\left(\hat{Y}_{L P M}\right)=\frac{1}{2} \sum_{i \in S}\left(\frac{y_{i}}{\pi_{i}}-\frac{y_{j_{i}}}{\pi_{j_{i}}}\right)^{2}
$$

where $j_{i}$ is the nearest neighbor to $i$ in the realized sample, i.e. the nearest neighbor in terms of the

157 auxiliary space in which the sample is balanced (see Grafström and Schelin 2013). In addition a variance 
158 estimator by Deville (1993), which also estimates the variance of an estimator without second order

159 inclusion probabilities, was applied:

160

$$
\widehat{V}_{D}\left(\hat{Y}_{L P M}\right)=\frac{1}{1-\sum_{i \in S} a_{i}^{2}} \sum_{i \in S}\left(1-\pi_{i}\right)\left(\frac{y_{i}}{\pi_{i}}-\sum_{j \in S} a_{j} \frac{y_{j}}{\pi_{j}}\right)^{2}
$$

161

Where $a_{i}=\left(1-\pi_{i}\right) / \sum_{j \epsilon s}\left(1-\pi_{j}\right)$ and $s$ refers to the selected sample. For variance estimation of the baseline designs (SI and 2PS) we refer to standard estimators provided in textbooks (e.g., Särndal et al. 1992, p. 353 for 2PS and p. 68 for SI).

\section{Simulated damage populations}

165

Damage populations were simulated with different spatial patterns and damage intensities to resemble damage caused by the spruce bark beetle (Ips typographus) at different intensities. The forest landscape was simulated using kNN-Sweden (Reese et al. 2003). First, two populations were simulated: one where damage incidence is highly dependent on spruce volume (IPS), and another where this dependence is less strong and the incidence of damage is less frequent (IPSALT). Details of these populations are described in Table 1 and Roberge et al. (2016). Two additional populations were introduced to cover

171 important cases with a spatial trend in the number of damaged trees in affected pixels, with lower

172 intensities of damage trees towards the edges of the municipality for IPSS and IPSALTS. These new 173 damage populations were based on new realizations of IPS and IPSALT. Using the following functions, 174 the frequency of damage trees was made more concentrated in the center of a chosen epicenter and 175 decrease away from it:

$$
f(x, y)=g(x) * g(y)
$$

177 Where $x$ and $y$ are the center coordinate of each grid-cell, and

$$
g(x)=1-\left(\frac{|x-w x|}{l x}\right)
$$

179 where $w x$ was set to 1600000 , a coordinate within the AOI, and $l x=x_{\max }-x_{\min }$ 
180

181

182

183

184

185

186

187

$$
g(y)=1-\left(\frac{|y-w y|}{2 l y}\right)
$$

where $w y$ was set to 7035000 , a chosen coordinate within the AOI and $l y=y_{\max }-y_{\min }$.

Eq. 7 returns values between 0.29 and 1 for each grid-cell (i.e. for each grid-cell coordinate $x$ and $y$ ) creating a diamond shaped area with the highest numbers close to the chosen center coordinates. The values from this raster layer were multiplied with the realized numbers of damaged trees in the baseline scenarios described by IPS and IPSALT (Roberge et al. 2016). The results (in terms of number of damage trees) were rounded upwards. The resulting damage layer has higher damage tree counts near the epicenter (wx, wy).

\section{Simulated auxiliary data}

DTC data layers were simulated with different errors. They had detection and false-detection probabilities equal for the whole municipality as described in Roberge et al. (2016) for the IPS scenarios.

These data layers correspond to classified remote sensing (RS) data, or the results of photo interpretation or helicopter inventory. In this study, however, we used only the data available for the first-phase grid-cells. The different combinations of detection probabilities and commission errors were named with a number corresponding to the detection probability $(90 \%$, etc.) and a suffix providing the level of commission error ( $\mathrm{L}$ for 0.001 and $\mathrm{H}$ for 0.05 ). Hence, DTC90L and DTC9OH refer to $90 \%$ detection and the lowest and highest probabilities of commission error, respectively.

\section{Monte Carlo Simulations}

For comparing the sampling strategies, Monte Carlo simulation was applied in the AOI for the different populations. All estimators that we used are unbiased for the population totals (Table 1 ) and thus the mean of estimated values approaches the true totals as the number of repetitions increases. The empirical relative standard deviation (ERSD) of the estimators was calculated as:

$$
\operatorname{ERSD}(\hat{Y})=\frac{\sqrt{\frac{1}{M} \sum_{m=1}^{M}\left(\hat{Y}_{m}-Y\right)^{2}}}{Y}
$$


203 Where $Y$ is the true population total and $\widehat{Y}_{m}$ is the estimated value from the $m$ :th repetition of the $M$ 204 repetitions. In order to evaluate the variance estimators we also calculated the ratio $(R)$ of the empirical mean of each variance estimator to the empirical variance of the estimator:

206

$$
R=\frac{\frac{1}{M} \sum_{m=1}^{M} \widehat{V}\left(\hat{Y}_{L P M}\right)_{m}}{\frac{1}{M} \sum_{m=1}^{M}\left(\hat{Y}_{m}-Y\right)^{2}}
$$

Simulations, analysis and visualizations were made with R (R Development Core Team 2016), using the R packages raster (Hijmans 2014), rgdal (Bivand et al. 2014), rasterVis (Perpinan Lamiguero and Hijmans 2014), data.table (Dowle et al. 2014), ggplot2 (Wickham 2009), sampling (Tillé and Matei 2014) and BalancedSampling (Grafström and Lisic 2016).

\section{Results}

212 LPM resulted in a more even ERSD (across the different populations, auxiliary data, and sample sizes)

213 compared to the 2PS design, and the ERSD for LPM was consistently lower than the ERSD for the SI

214 design (figure 2 and 3, table 2). For good auxiliary data with appropriate allocation, the 2PS design

215 resulted in the lowest ERSD.

216 ERSD from SI sampling in IPS was close to the ERSD from SI sampling in IPSS. Only at small sample sizes

217 did the slightly smaller population variance of the IPSS lead to a slight difference in the ERSD when the

218 two were compared for SI design. The other "twin" populations (IPSALT and IPSALTS) were also similar in

219 ERSD and followed a similar pattern.

220 LPM applied to the two populations with most damage (IPS and IPSS) also resulted in similar ERSD, i.e.

221 the ERSD from LPM sampling in IPS only differed slightly from the ERSD from LPM sampling in IPSS, with

222 the same sample size and auxiliary data. LPM and the best auxiliary data scenario resulted in lower ERSD

223 from sampling the IPSS population relative to the IPS population with the same estimator and auxiliary

224 data for all sample sizes. The same pattern was found for the LPM design in IPSALT and IPSALTS: again, 
225 the ERSD was lower for accurate auxiliary data and for the population with spatial trend, and for the 226 population with lower true population variance.

227 For the 2PS design, there was a large difference in ERSD between the different types of auxiliary data.

228 The best auxiliary data resulted in large improvements of the ERSD relative to the worst auxiliary data.

229 With poor auxiliary data the ERSD was higher than the ERSD of the LPM, and in some cases SI performed

230 almost as well as 2PS. For a different, more even allocation, the ERSD from 2PS was lower, but the

231 accuracy of the auxiliary data still made a difference. The improvement with better auxiliary data was

232 more pronounced in the IPSS and IPSALTS damage population scenarios

233 The Deville estimator $\left(\widehat{V}_{D}\right)$ for $\widehat{Y}_{L P M}$ overestimated the variance (Table 3). The ratio $(R)$ was 1.7-4.7

234 compared to the empirical variance. The Grafström-Schelin variance estimator $\left(\widehat{V}_{G S}\right)$ worked very well in

235 all scenarios and auxiliary data combinations, with a mean estimated variance to empirical variance ratio $236(R)$ of approximately 1. 


\section{Discussion}

239 Generally, aerial detection surveys or aerial photo interpretation of damage over affected areas need to

240 be performed when the damage is visible, but preferably before it has had time to spread further

241 (Backsen and Howell 2013). This is the case also when other kinds of remote sensing data are used (e.g.

242 Hall et al. 2016). In forest damage inventories, especially when a new outbreak has been noted and

243 there is a need to acquire information for decisions on, and support for, potential mitigation strategies,

244 the time available for setting up the inventory is often limited and the inventory needs to be performed

245 quickly (e.g. Backsen and Howell 2013). For efficient mitigation of bark beetle outbreaks it is important

246 to quickly localize affected trees, while the beetles of the next generation are still under the bark of the

247 recently killed trees (Lindelöw \& Schroeder 2008). These trees are often called "green attack trees", and

248 are difficult to detect accurately at this stage (Wulder et al 2009). Remotely sensed data about green

249 attack trees, or data on forest vitality from remote sensing, may not be appropriate for direct

250 application in mitigation schemes (e.g. Lausch et al. 2013). However, such data may be useful for

251 efficiently spreading the sample for a forest damage inventory, especially since it is possible to select a

252 sub-sample from a first-phase sample of auxiliary data (e.g. utilizing high spatial and spectral resolution

253 data obtained for a sample of the AOI). Since the correlation between this type of data and actual bark

254 beetle damage may be low, utilization in 2PS sampling may not be efficient due to poor classification

255 accuracy (Figure 2 and 3). However, the LPM is less sensitive to such problems since the method does

256 not rely on using the auxiliary information for classification but only for obtaining an efficiently spread

257 sample in the space of the auxiliary variables. Using the LPM it is possible to utilize other types of

258 auxiliary data as well, such as map layers with estimated spruce volume, which may be correlated with

259 spruce bark beetle damage (Kärvemo et al. 2014). Such combinations of auxiliary data contribute to

260 efficiently spread the sample using LPM and thus result in improved estimates. 
261 The spruce bark beetle is one of the most important forest pests in Europe (Schelhaas, Naaburs \& 262 Schuck 2003), and the most important damaging insect on Norway spruce in northern Europe (Weslien 263 1992). For example, it killed approximately 3 million $\mathrm{m}^{3}$ of spruce in southern Sweden following two 264 large storms in 2005-2006, causing additional losses for many forest owners (Långström et al. 2010). In 265 such a situation it may be important to add as small additional costs as possible to the losses already 266 incurred, and thus it could be important to keep inventory costs low while still obtaining relevant 267 information for the mitigation activities (e.g. Herrick 1981).

268 Studying the results obtained from the 2PS design, there was a large difference between the ERSDs from 269 using different kinds of auxiliary data and, more noticeably, there was a large difference in the ERSD of 270 the estimator depending on the allocation of the second phase sample. Poor auxiliary data resulted in 271 higher ERSD, especially for the scenarios where there was a spatial trend in the damage intensity of the

272 sampled population (i.e. IPSS and IPSALTS). This is interesting since these populations had lower

273 population variance in comparison to their twin versions. Hence, these results in terms of ERSD were 274 solely dependent on the spatial difference between the two populations. This volatility was especially 275 pronounced in scenarios where a large part of S2 was allocated to the stratum with detected damage 276 trees. However, when the auxiliary information was good and the allocation adequate, 2PS 277 outperformed LPM in terms of ERSD.

278 While not pursued in this study, the performance of LPM might be improved upon if the auxiliary 279 information is used for modifying the inclusion probabilities in LPM (e.g. two-phase for stratification 280 LPM sampling, or by letting the inclusion probabilities be proportional to the numbers of damage trees 281 in the auxiliary data). In this case it is likely that LPM would have outperformed 2PS with STSI. However, 282 our application of LPM was straightforward and it is interesting to see how much it improved the 283 precision of the estimates compared to the SI design. 
284 In general, this study resulted in higher ERSDs than those obtained in Roberge et al. (2016), where 2PS

285 was used as well. However, an important difference is that in the current study we did not assume any 286 prior knowledge of the domain of interest (i.e. the population susceptible to the damage); in Roberge et 287 al (2016) such information was available from previously surveyed national forest inventory field plots. 288 Also, Roberge et al. (2016) showed that wall-to-wall remotely sensed data and post-stratification 289 improved the precision of estimates further.

290 For variance estimation, two different variance estimators were applied to the LPM-estimator. The

291 Deville estimator for $\widehat{Y}_{L P M}$ overestimated the variance (table 3), while the Grafström-Schelin variance 292 estimator worked very well in all scenarios and auxiliary data combinations. The Deville estimator 293 assumes a high level of randomness in the sample selection and thus worked well for SI samples.

294 However, it does not exploit the induced stratification effect by the LPM which results in a conservative 295 variance estimation for such designs. The Grafström-Schelin variance estimator is tailored for well296 spread samples and has previously been shown to sometimes slightly overestimate the variance for 297 large samples for volume estimates (Grafström and Ringvall, 2013).

298 Results from LPM stand out as consistently better then SI, and relatively unaffected by different kinds of 299 auxiliary data when compared with the 2PS design. In addition, there is great practical advantage in 300 avoiding the division into strata and the decision on sample allocation between strata - these issues can 301 be avoided if the LPM is applied. The two-phase design was used in order to speed up the sample 302 selection with the LPM algorithm. In this study there was no difference in the precision depending on 303 the size of the first phase sample. However, it can be noted that large first-phase samples would 304 increase the cost of LPM and 2PS relative to the cost of SI where only the cost of the field-campaign is 305 incurred. This way of spreading the sample, applying the LPM in a two-phase strategy, may be indicative 306 of the competitiveness of LPM in continuous sample selection or for application to larger areas. With 
307 this two-phase application it could be used in a continuous approach where unprocessed data from

308 point clouds (i.e. from ALS or photogrammetric matching of digital aerial images) can be utilized in a first 309 phase. so while the LPM could utilize the unclassified data space from remotely sensed data together 310 with other auxiliary GIS-information that may be correlated with the damage event of interest (e.g. GIS

311 layers of elevation, temperature or precipitation) to select a balanced sample, the 2PS needs processed 312 data with readily available classifications for the stratification. I.e. the LPM could potentially use data 313 with less processing to select a sample that is balanced in the auxiliary space, and hence there is less

314 need for extensive data processing/modeling or classification procedures in order to utilize the

315 information for designing the inventory than would be the case for applying, for instance, STSI or 2PS

316 sampling designs. In addition to practicality, auxiliary data spaces from different remote sensing

317 techniques could be much better than what was examined here, containing more information than the

318 classified data we used in this study. If for instance the variable to estimate had been the damaged

319 volume, first return data or highest point-cloud information would have been correlated with the

320 volume, thus further improving the LPM's performance.

321 Overall, 2PS sampling is a competitive and efficient means of utilizing auxiliary information in selection.

322 With good auxiliary information, it is possible to choose a good allocation and sample 2PS with good

323 results, but this may be difficult when the information is flawed, and its true state is unknown.

324 For variance estimation it is advisable to utilize the $\widehat{V}_{G S}$ estimator (eq. 5).

325 In conclusion the LPM has been shown to be a better choice than SI in all cases evaluated. Considering 326 that more auxiliary data sources could potentially be utilized, LPM stands out as a straightforward 327 approach to use such data to improve forest damage inventories. 


\section{Acknowledgements}

329 We wish to thank two anonymous reviewers for their valuable comments that helped us to improve the 330 manuscript. We also wish to thank Sören Wulff and the staff of the Swedish NFI and TFDI for their 331 valuable work contributing to building the simulated populations. Cornelia Roberge was supported by 332 grant 2008-546 from the Swedish Research Council Formas to Anna Hedström-Ringvall.

333

334

335

336

337

338

339

340

341

342

343

344

345

346

347

348

349

350

\section{References}

Allen, C. D., et al. 2010. A global overview of drought and heat-induced tree mortality reveals emerging climate change risks for forests. Forest Ecology and Management 259(4): 660-684.

Aamlid, D., et al. 2000. Changes of forest health in Norwegian boreal forests during 15 years. Forest Ecology and Management 127(1-3): 103-118.

Backsen, J. C. and Howell, B. 2013. Comparing aerial detection and photo interpretation for conducting forest health surveys. Western Journal of Applied Forestry 28(1): 3-8.

Bennett, D. D. and Tkacz, B. M. 2008. Forest health monitoring in the United States: a program overview. Australian Forestry. 71(3): 223-228.

Bivand, R., Keitt, T. and Rowlingson B. 2016. rgdal: Bindings for the Geospatial Data Abstraction Library. R package version 1.1-10. https://CRAN.R-project.org/package=rgdal

\footnotetext{
Carnegie, A. J. 2008. A decade of forest health surveillance in Australia: an overview. Australian Forestry.
} 71(3): 161-163. 
352 Ciesla, W.M. 2000. Remote sensing in forest health protection (FHTET Report No. 00-03). Retrieved from www.fs.fed.us/foresthealth/technology/pdfs/RemoteSensingForestHealth00_03.pdf $(13: 17,2016-09-06)$

Corbett, L. J., Withey, P., Lantz, V. A., \& Ochuodho T. O. 2015. The economic impact of the mountain pine beetle infestation in British Columbia: provincial estimates from a CGE analysis. Forestry. doi: $10.1093 /$ forestry/cpv042

359

Dale, V. H., Joyce, L. A., McNulty, S., Neilson, R. P., Ayres, M. P., Flannigan, M. D., et al. 2001. Climate Change and Forest Disturbances: Climate change can affect forests by altering the frequency, intensity, duration, and timing of fire, drought, introduced species, insect and pathogen outbreaks, hurricanes, windstorms, ice storms, or landslides. BioScience, 51(9), 723-734, doi:10.1641/0006-3568(2001)051[0723:ccafd]2.0.co;2.

Deville, J. C. 1993. Estimation de la variance pour les enquêtes en deux phases. Manuscript. Paris, France: INSEE.

Dowle, M., Srinivasan, A., Short, T., Lianoglou S., with contributions from Saporta, R., and Antonyan, E. 2015. data.table: Extension of Data.frame. R package version 1.9.6. https://CRAN.R- 
373 Ferretti, M., König, N., Rautio, P., \& Sase, H. 2009. Quality assurance (QA) in international forest

374

375

376

377

378

379

380

381

382

383

384

385

386

387

388

389

390

391

392

393

394

395 monitoring programmes: activity, problems and perspectives from East Asia and Europe. Annals of Forest Science, 66(4), 1-12.

Grafström, A. and Lisic, J. 2016. BalancedSampling: Balanced and Spatially Balanced Sampling. R package version 1.5.2. https://CRAN.R-project.org/package=BalancedSampling

Grafström, A., and Lundström, N.L.P. 2013. Why well spread probability samples are balanced. Open Journal of Statistics, 3(1): 36-41. doi:10.4236/ojs.2013.31005.

Grafström, A., and Ringvall, A.H. 2013. Improving forest field inventories by using remote sensing data in novel sampling designs. Can. J. For. Res. 43(11):1015-1022. doi: 10.1139/cjfr-2013-0123.

Grafström, A., and Schelin, L. 2013. How to select representative samples. Scand.J. Stat. 41(2): 277-290. doi: 10.1111/sjos.12016.

Grafström, A., Lundström, N.L.P., and Schelin, L. 2012. Spatially balanced sampling through the Pivotal method. Biometrics, 68(2): 514-520. doi: 10.1111/j.1541-0420.2011.01699.x.

Grafström, A., Saarela, S., \& Ene, L. T. 2014. Efficient sampling strategies for forest inventories by spreading the sample in auxiliary space. Canadian Journal of Forest Research, 44(10), 11561164. 
401

402

403

404

405

406

407

408

409

410

411

412

Hall, R. J., et al. 2016. Remote sensing of forest pest damage: a review and lessons learned from a Canadian perspective*. The Canadian Entomologist: 1-61.

Hais, M., et al. 2009. Comparison of two types of forest disturbance using multitemporal Landsat TM/ETM+ imagery and field vegetation data. Remote Sensing of Environment 113(4): 835-845.

Herrick, O. W. (1981). Forest Pest Management Economics--Application to the Gypsy Moth. Forest Science, 27(1), 128-138.

Hijmans, Robert J. (2016). raster: Geographic Data Analysis and Modeling. R package version 2.5-8. https://CRAN.R-project.org/package=raster

Kärvemo, S., Van Boeckel, T. P., Gilbert, M., Grégoire, J.-C. \& Schroeder, M. (2014). Large-scale risk mapping of an eruptive bark beetle - Importance of forest susceptibility and beetle preassure. Forest Ecology and Management. 318:158-166.

Långström, B., Lindelöw, Å., Schroeder, M., Björklund, N. \& Öhrn, P. (2010). The spruce bark beetle outbreak in Sweden following the january-storms in 2005 and 2007. Available from: http://pub.epsilon.slu.se/5076/1/langstrom_b_etal_100823.pdf. [2016-10-20]

Lausch, A., Heurich, M., Gordalla, D., Dobner, H.-J., Gwillym-Margianto, S. and Salbach, C. (2013). Forecasting potential bark beetle outbreaks based on spruce forest vitality using hyperspectral remote-sensing techniques at different scales. Forest Ecology and Management 308:76-89. 
420

421

422

423

424

425

426

427

428

429

430

431

432

433

434

435

436

437

438

439

440

441

442

443

Lindelöw, Å. \& Schroeder, M. (2008). The storm "Gudrun" and the spruce bark beetle in Sweden. In: Steyrer, G., et al. (Eds.) Second meeting of Forest Protection and Forest Phytosanitary Specialists, Vienna, Austria, 27-28 November 2007. Forstschutz aktuell nr 44, Bundesforschungsund Ausbildungszentrum für wald, Naturgefahren und Landschaft (BFW), Vienna, Austria.

Mery, G., Alfaro, R.I., Kanninen, M., and Lobovikov, M. 2005. Forests in the global balance -changing paradigms. IUFRO World Series, Volume 17. International Union of Forestry Research Organizations (IUFRO), Vienna, Austria. Retrieved from: http://www.iufro.org/science/special/wfse/forests-society-global-drivers/ (2016-09-07 at 04:57)

Näsi, R., et al. (2015). Using UAV-Based Photogrammetry and Hyperspectral Imaging for Mapping Bark Beetle Damage at Tree-Level. Remote Sensing 7(11): 15467-15493.

Ortiz, S.M., Breidenbach, J., Kändler, G. 2013. Early Detection of Bark Beetle Green Attack Using TerraSAR-X and RapidEye Data. Remote Sens. 2013, 5, 1912-1931.

Perpinan Lamigueiro, O., and Hijmans, R., 2016, meteoForecast. R package version 0.40.

R Core Team (2016). R: A language and environment for statistical computing. R Foundation for Statistical Computing, Vienna, Austria. URL https://www.R-project.org/. 
445 Roberge, C., Wulff, S., Reese, H., \& Ståhl, G. 2016. Improving the precision of sample-based forest 446 damage inventories through two-phase sampling and post-stratification using remotely sensed auxiliary information. Environmental Monitoring and Assessment, 188(4), 1-21. doi: $10.1007 / \mathrm{s} 10661-016-5208-4$

449

450

Rullan-Silva, C. D., Olthoff, A. E., Delgado de la Mata, J. A. \& Pajares-Alonso J. A. (2013). Remote monitoring of forest insect defoliation. A review. Forest Systems 2013 22(3): 377-391

452

453

Schelhaas, M.-J., Nabuurs, G.-J. \& Schuck, A. (2003) Natural disturbances in the European forests in the 19th and 20th centuries. Global change biology, 9: 1620-1633. doi:10.1046/j.1365-

455 2486.2003.00684.x

456

457

Solberg, S. 2010. Mapping gap fraction, LAI and defoliation using various ALS penetration variables. International Journal of Remote Sensing 31(5): 1227-1244.

459

460

Särndal, C.-E., Swensson, B., \& Wretman, J. H. 1992. Model assisted survey sampling. New York: Springer-Verlag.

462

463

Tillé, Y., and Matei, A. 2015. sampling: Survey Sampling. R package version 2.7. https://CRAN.R-project.org/package=sampling

465

466

Trumbore, S., Brando, P. and Hartmann, H. (2015) Forest health and global change. Science.

467 349(6250):814-818. 
469 Weslien, J. (1992), Monitoring Ips typographus (L.) populations and forecasting damage1. Journal of 470 Applied Entomology, 114: 338-340. doi:10.1111/j.1439-0418.1992.tb01136.x

471

472 Wickham, H. 2009. ggplot2: Elegant Graphics for Data Analysis. Springer-Verlag, New York, 2009.

473

474 Wulder, M.A., White, J.C., Carroll, A.L. and Coops N.C. (2009). Challenges for the operational detection 475 of mountain pine beetle green attack with remote sensing. The Forestry Chronicle 85(1):32-38.

476

477 Wulff, S., Lindelöw, Å., Lundin, L., Hansson, P., Axelsson, A.-L., Barklund, P., Wijk, S., Ståhl, G. 2012.

$478 \quad$ Adapting forest health assessments to changing perspectives on threats - a case example from 479 Sweden. Environmental Monitoring and Assessment, 184(4), 2453-2464. doi: 10.1007/s10661480 011-2130-7 
482 Table 1. Number of damage trees $(y)$ and affected grid-cells $(1 / 0)$ in the simulated populations.

\begin{tabular}{|c|c|c|c|c|c|c|c|c|}
\hline & \multicolumn{2}{|c|}{ IPS } & \multicolumn{2}{|c|}{ IPSS } & \multicolumn{2}{|c|}{ IPSALT } & \multicolumn{2}{|c|}{ IPSALTS } \\
\hline & $1 / 0$ & $y$ & $1 / 0$ & $y$ & $1 / 0$ & $y$ & $1 / 0$ & $y$ \\
\hline True Total & $282 \times 10^{3}$ & $846 \times 10^{3}$ & $282 \times 10^{3}$ & $662 \times 10^{3}$ & $167 \times 10^{3}$ & $502 \times 10^{3}$ & $171 \times 10^{3}$ & $396 \times 10^{3}$ \\
\hline Mean & 0.20 & 0.59 & 0.20 & 0.46 & 0.12 & 0.35 & 0.12 & 0.27 \\
\hline$\sigma$ & 0.40 & 1.34 & 0.40 & 1.05 & 0.32 & 1.07 & 0.32 & 0.83 \\
\hline Maximum & 1 & 12 & 1 & 10 & 1 & 12 & 1 & 10 \\
\hline Minimum & 0 & 0 & 0 & 0 & 0 & 0 & 0 & 0 \\
\hline
\end{tabular}

483

484 
Table 2. Empirical relative standard deviations in \% over 1000 repetitions.

\begin{tabular}{|c|c|c|c|c|c|c|c|c|c|c|}
\hline \multirow[b]{2}{*}{ Population } & \multirow[b]{2}{*}{ Aux.data } & \multirow[b]{2}{*}{ Design } & \multicolumn{4}{|c|}{$n_{1}=\mathbf{3 6 0 0}$} & \multicolumn{4}{|c|}{$n_{1}=10000$} \\
\hline & & & $n_{2}=50$ & $n_{2}=100$ & $n_{2}=150$ & $n_{2}=\mathbf{2 0 0}$ & $n_{2}=50$ & $n_{2}=100$ & $n_{2}=150$ & $n_{2}=\mathbf{2 0 0}$ \\
\hline \multirow[t]{12}{*}{ IPS } & DTC60H & LPM & 22 & 16 & 13 & 12 & 23 & 16 & 13 & 11 \\
\hline & & 2PS_40 & 21 & 15 & 12 & 10 & 22 & 15 & 12 & 11 \\
\hline & & 2PS_20 & 25 & 19 & 16 & 13 & 25 & 19 & 15 & 13 \\
\hline & DTC60L & LPM & 20 & 14 & 11 & 10 & 20 & 14 & 11 & 10 \\
\hline & & 2PS_40 & 20 & 13 & 11 & 10 & 20 & 13 & 11 & 10 \\
\hline & & 2PS_20 & 26 & 18 & 15 & 13 & 25 & 17 & 14 & 13 \\
\hline & DTC90H & LPM & 20 & 14 & 12 & 10 & 21 & 14 & 11 & 10 \\
\hline & & 2PS_40 & 15 & 10 & 9 & 7 & 15 & 11 & 9 & 7 \\
\hline & & 2PS_20 & 15 & 11 & 9 & 8 & 15 & 10 & 8 & 7 \\
\hline & DTC90L & LPM & 18 & 12 & 10 & 9 & 18 & 12 & 10 & 8 \\
\hline & & 2PS_40 & 11 & 8 & 7 & 6 & 11 & 8 & 6 & 6 \\
\hline & & 2PS_20 & 11 & 9 & 7 & 6 & 12 & 9 & 7 & 6 \\
\hline \multirow[t]{12}{*}{ IPSS } & DTC60H & LPM & 23 & 17 & 14 & 12 & 23 & 16 & 14 & 12 \\
\hline & & 2PS_40 & 22 & 16 & 13 & 11 & 22 & 15 & 13 & 11 \\
\hline & & 2PS_20 & 31 & 21 & 18 & 14 & 29 & 21 & 16 & 14 \\
\hline & DTC60L & LPM & 20 & 13 & 11 & 10 & 19 & 13 & 11 & 9 \\
\hline & & 2PS_40 & 21 & 15 & 12 & 11 & 21 & 15 & 12 & 11 \\
\hline & & 2PS_20 & 30 & 21 & 16 & 15 & 29 & 19 & 17 & 14 \\
\hline & DTC90H & LPM & 20 & 14 & 11 & 10 & 21 & 14 & 11 & 10 \\
\hline & & 2PS_40 & 15 & 11 & 9 & 8 & 16 & 11 & 9 & 8 \\
\hline & & 2PS_20 & 16 & 11 & 9 & 8 & 16 & 11 & 9 & 8 \\
\hline & DTC90L & LPM & 17 & 11 & 9 & 8 & 17 & 11 & 9 & 8 \\
\hline & & 2PS_40 & 12 & 8 & 7 & 6 & 11 & 8 & 6 & 6 \\
\hline & & 2PS_20 & 13 & 9 & 8 & 7 & 12 & 9 & 8 & 7 \\
\hline \multirow[t]{12}{*}{ IPSALT } & DTC60H & LPM & 32 & 23 & 19 & 16 & 33 & 23 & 18 & 16 \\
\hline & & 2PS_40 & 30 & 21 & 16 & 14 & 29 & 20 & 17 & 14 \\
\hline & & 2PS_20 & 37 & 23 & 20 & 18 & 34 & 25 & 20 & 17 \\
\hline & DTC60L & LPM & 26 & 18 & 15 & 13 & 28 & 18 & 15 & 13 \\
\hline & & 2PS_40 & 25 & 18 & 15 & 13 & 25 & 17 & 15 & 13 \\
\hline & & 2PS_20 & 33 & 24 & 20 & 17 & 33 & 25 & 20 & 17 \\
\hline & DTC90H & LPM & 28 & 20 & 16 & 14 & 28 & 19 & 15 & 14 \\
\hline & & 2PS_40 & 19 & 14 & 12 & 10 & 19 & 14 & 11 & 10 \\
\hline & & 2PS_20 & 19 & 14 & 11 & 10 & 20 & 14 & 11 & 10 \\
\hline & DTC90L & LPM & 24 & 17 & 14 & 12 & 24 & 16 & 13 & 11 \\
\hline & & 2PS_40 & 13 & 11 & 9 & 8 & 12 & 9 & 8 & 6 \\
\hline & & 2PS_20 & 15 & 11 & 10 & 9 & 15 & 10 & 8 & 8 \\
\hline \multirow[t]{12}{*}{ IPSALTS } & DTC60H & LPM & 33 & 22 & 19 & 16 & 33 & 23 & 19 & 16 \\
\hline & & 2PS_40 & 30 & 20 & 18 & 16 & 33 & 22 & 18 & 15 \\
\hline & & 2PS_20 & 39 & 27 & 23 & 20 & 41 & 26 & 22 & 20 \\
\hline & DTC60L & LPM & 26 & 19 & 15 & 13 & 26 & 17 & 15 & 12 \\
\hline & & 2PS_40 & 28 & 21 & 16 & 14 & 30 & 19 & 18 & 14 \\
\hline & & 2PS_20 & 42 & 28 & 24 & 19 & 39 & 28 & 23 & 19 \\
\hline & DTC9OH & LPM & 28 & 20 & 16 & 14 & 27 & 20 & 16 & 14 \\
\hline & & 2PS_40 & 20 & 15 & 12 & 10 & 20 & 14 & 11 & 10 \\
\hline & & 2PS_20 & 20 & 16 & 13 & 10 & 21 & 14 & 11 & 10 \\
\hline & DTC90L & LPM & 23 & 15 & 12 & 10 & 22 & 15 & 12 & 10 \\
\hline & & 2PS_40 & 13 & 10 & 9 & 8 & 14 & 9 & 7 & 7 \\
\hline & & 2PS_20 & 19 & 12 & 11 & 10 & 16 & 11 & 11 & 8 \\
\hline
\end{tabular}

Note: ERSD below $10 \%$ are in bold text and above $25 \%$ are cursive. 
487 Table 3. Ratio of mean $\widehat{V}_{G S}\left(\hat{Y}_{L P M}\right)$ and mean $\widehat{V}_{D}\left(\hat{Y}_{L P M}\right)$ to empirical variance over 1000 repetitions.

\begin{tabular}{|c|c|c|c|c|c|c|c|c|c|c|}
\hline \multirow[b]{2}{*}{ Population } & \multirow[b]{2}{*}{ Aux.data } & & \multicolumn{4}{|c|}{$n_{1}=3600$} & \multicolumn{4}{|c|}{$n_{1}=10000$} \\
\hline & & & $n_{2}=50$ & $n_{2}=100$ & $n_{2}=150$ & $n_{2}=200$ & $n_{2}=50$ & $n_{2}=100$ & $n_{2}=150$ & $n_{2}=200$ \\
\hline \multirow[t]{8}{*}{ IPS } & DTC60H & $\widehat{V}_{G S}$ & 1.1 & 1.0 & 1.0 & 0.9 & 1.0 & 0.9 & 1.0 & 1.0 \\
\hline & & $\widehat{V}_{D}$ & 2.1 & 2.1 & 2.1 & 1.9 & 1.9 & 1.9 & 2.0 & 2.1 \\
\hline & DTC60L & $\widehat{V}_{G S}$ & 1.0 & 1.0 & 1.0 & 0.9 & 1.1 & 1.0 & 1.0 & 0.9 \\
\hline & & $\widehat{V}_{D}$ & 2.5 & 2.7 & 2.8 & 2.6 & 2.6 & 2.7 & 2.9 & 2.8 \\
\hline & DTC90H & $\widehat{V}_{G S}$ & 0.9 & 1.0 & 0.9 & 1.0 & 0.8 & 0.9 & 1.0 & 0.9 \\
\hline & & $\widehat{V}_{\boldsymbol{D}}$ & 2.6 & 2.9 & 2.6 & 2.7 & 2.5 & 2.7 & 2.8 & 2.5 \\
\hline & DTC90L & $\widehat{V}_{G S}$ & 1.0 & 1.0 & 1.0 & 1.0 & 1.0 & 1.1 & 1.0 & 1.1 \\
\hline & & $\hat{V}_{D}$ & 3.3 & 3.5 & 3.3 & 3.6 & 3.2 & 3.7 & 3.6 & 4.1 \\
\hline \multirow[t]{8}{*}{ IPSSS } & DTC60H & $\widehat{V}_{G S}$ & 1.0 & 1.0 & 1.0 & 0.9 & 1.1 & 1.0 & 1.0 & 0.9 \\
\hline & & $\widehat{V}_{D}$ & 1.9 & 1.8 & 1.9 & 1.8 & 1.9 & 1.9 & 1.9 & 1.8 \\
\hline & DTC60L & $\widehat{V}_{G S}$ & 1.1 & 1.0 & 0.9 & 0.9 & 1.1 & 1.1 & 1.0 & 1.0 \\
\hline & & $\widehat{V}_{\boldsymbol{D}}$ & 2.8 & 2.9 & 2.7 & 2.7 & 2.8 & 2.9 & 2.7 & 2.9 \\
\hline & DTC9OH & $\widehat{V}_{G S}$ & 1.0 & 0.9 & 1.0 & 1.0 & 0.9 & 1.0 & 1.0 & 1.0 \\
\hline & & $\hat{V}_{\boldsymbol{D}}$ & 2.6 & 2.6 & 2.7 & 2.8 & 2.5 & 2.7 & 2.9 & 2.7 \\
\hline & DTC90L & $\widehat{V}_{G S}$ & 1.1 & 1.1 & 1.0 & 0.9 & 1.2 & 1.1 & 1.0 & 1.0 \\
\hline & & $\widehat{V}_{D}$ & 3.5 & 4.2 & 4.1 & 4.1 & 3.8 & 4.1 & 4.3 & 4.4 \\
\hline \multirow[t]{8}{*}{ IPSALT } & DTC60H & $\widehat{V}_{G S}$ & 1.0 & 0.9 & 0.9 & 1.0 & 1.0 & 0.9 & 1.0 & 1.0 \\
\hline & & $\hat{V}_{\boldsymbol{D}}$ & 1.9 & 1.8 & 1.8 & 1.9 & 1.8 & 1.7 & 1.9 & 1.9 \\
\hline & DTC60L & $\widehat{V}_{G S}$ & 1.1 & 1.0 & 1.0 & 0.9 & 1.0 & 1.1 & 0.9 & 0.9 \\
\hline & & $\hat{V}_{\boldsymbol{D}}$ & 2.9 & 2.8 & 2.8 & 2.9 & 2.5 & 3.0 & 2.8 & 2.9 \\
\hline & DTC9OH & $\widehat{V}_{G S}$ & 0.9 & 0.9 & 0.9 & 0.9 & 0.9 & 1.0 & 1.0 & 0.9 \\
\hline & & $\widehat{V}_{\boldsymbol{D}}$ & 2.5 & 2.5 & 2.5 & 2.4 & 2.5 & 2.6 & 2.7 & 2.5 \\
\hline & DTC90L & $\widehat{V}_{G S}$ & 1.0 & 0.9 & 0.9 & 0.9 & 1.0 & 1.1 & 1.0 & 1.0 \\
\hline & & $\hat{V}_{D}$ & 3.4 & 3.2 & 3.3 & 3.5 & 3.3 & 3.7 & 3.8 & 3.9 \\
\hline \multirow[t]{8}{*}{ IPSALTS } & DTC60H & $\widehat{V}_{G S}$ & 1.0 & 1.0 & 1.0 & 1.0 & 1.0 & 1.0 & 1.0 & 1.1 \\
\hline & & $\hat{V}_{D}$ & 1.7 & 1.8 & 1.7 & 1.7 & 1.7 & 1.7 & 1.7 & 1.9 \\
\hline & DTC60L & $\hat{V}_{G S}$ & 1.1 & 0.9 & 0.9 & 0.9 & 1.2 & 1.1 & 1.0 & 1.0 \\
\hline & & $\hat{V}_{D}$ & 2.7 & 2.5 & 2.5 & 2.7 & 2.9 & 3.1 & 2.9 & 3.0 \\
\hline & DTC9OH & $\widehat{V}_{G S}$ & 0.9 & 1.0 & 1.0 & 0.9 & 1.1 & 1.0 & 1.0 & 1.0 \\
\hline & & $\hat{V}_{D}$ & 2.3 & 2.4 & 2.4 & 2.3 & 2.6 & 2.4 & 2.5 & 2.5 \\
\hline & DTC90L & $\hat{V}_{G S}$ & 1.0 & 1.0 & 1.0 & 1.0 & 1.1 & 1.1 & 1.0 & 1.1 \\
\hline & & $\widehat{V}_{D}$ & 3.6 & 4.0 & 4.2 & 4.3 & 3.9 & 4.3 & 4.5 & 4.7 \\
\hline
\end{tabular}


490 Figure 1. $900 \mathrm{~km}^{2}$ landscape with simulated true numbers of damage trees for the four damage

491 populations a. IPS, b. IPSS, c. IPSALT, d. IPSALTS and e. true spruce volume layer from which damage

492 were simulated.

493

494 Figure 2. ERSD for $n_{1}=10000$ and all combinations of damage, sample size, sampling strategy and 495 auxiliary information used. ERSD for SI is included as a straight line.

496

497 Figure 3. ERSD for $\boldsymbol{n}_{\mathbf{1}}=3600$, and all combinations of damage, sample size, sampling strategy and 498 auxiliary information used. ERSD from SI design in straight line across. 


\section{Appendices}

501

502 Table A1. ERSD of SI for each damage scenario and sample size.

\begin{tabular}{llrrrr}
\hline & Sample size & 50 & 100 & 150 & 200 \\
\hline Damage & IPS & $32 \%$ & $23 \%$ & $18 \%$ & $16 \%$ \\
& IPSALT & $45 \%$ & $30 \%$ & $24 \%$ & $21 \%$ \\
& IPSALTS & $43 \%$ & $29 \%$ & $25 \%$ & $22 \%$ \\
& IPSS & $33 \%$ & $23 \%$ & $18 \%$ & $16 \%$ \\
\hline
\end{tabular}


Table A2. Actual sensitivity of simulated auxiliary spaces - True positive rates (ie. DTC 1-1,2-2,3-3 etc, but for D: 1-1/0-0))

\begin{tabular}{llll}
\hline $\begin{array}{l}\text { Damage } \\
\text { Population }\end{array}$ & Auxiliary data & $\begin{array}{l}\text { Sensitivity } \\
\text { (exact DTC) }\end{array}$ & $\begin{array}{l}\text { Sensitivity } \\
\text { (D) }\end{array}$ \\
\hline IPS & DTC90L & 0.925 & 0.983 \\
IPS & DTC9OH & 0.925 & 0.983 \\
IPS & DTC60L & 0.604 & 0.880 \\
IPS & DTC60H & 0.604 & 0.880 \\
IPSS & DTC90L & 0.919 & 0.974 \\
IPSS & DTC9OH & 0.920 & 0.975 \\
IPSS & DTC60L & 0.582 & 0.828 \\
IPSS & DTC6OH & 0.583 & 0.830 \\
IPSALT & DTC9OL & 0.925 & 0.983 \\
IPSALT & DTC9OH & 0.925 & 0.983 \\
IPSALT & DTC60L & 0.607 & 0.881 \\
IPSALT & DTC60H & 0.603 & 0.879 \\
IPSALTS & DTC90L & 0.923 & 0.975 \\
IPSALTS & DTC9OH & 0.923 & 0.975 \\
IPSALTS & DTC60L & 0.590 & 0.830 \\
IPSALTS & DTC60H & 0.586 & 0.829 \\
\hline
\end{tabular}



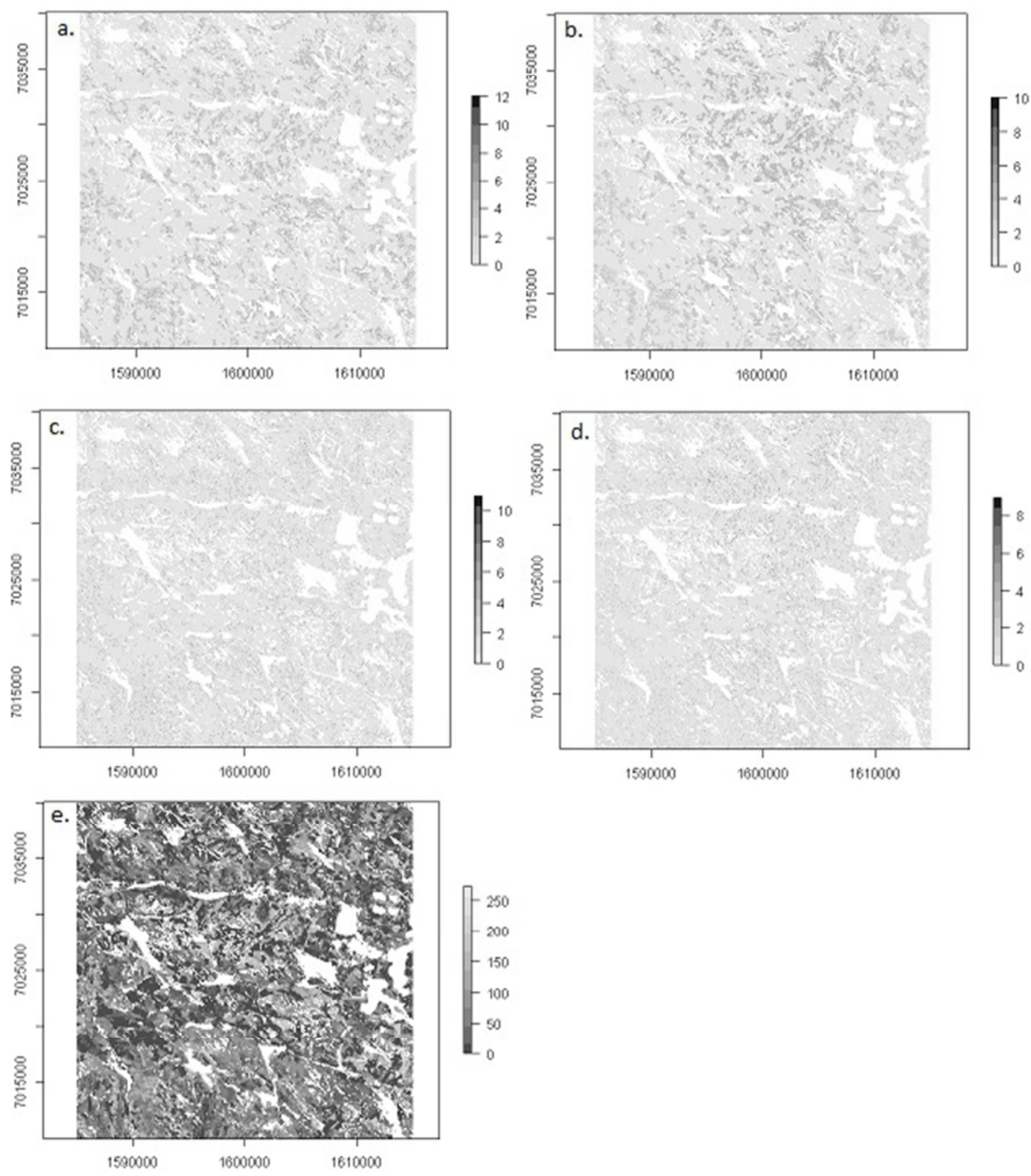

$900 \mathrm{~km} 2$ landscape with simulated true numbers of damage trees for the four damage populations $\mathrm{a}$. IPS, $\mathrm{b}$. IPSS, c. IPSALT, d. IPSALTS and e. true spruce volume layer from which damage were simulated.

Figure 1

$174 \times 207 \mathrm{~mm}$ (96 x 96 DPI) 


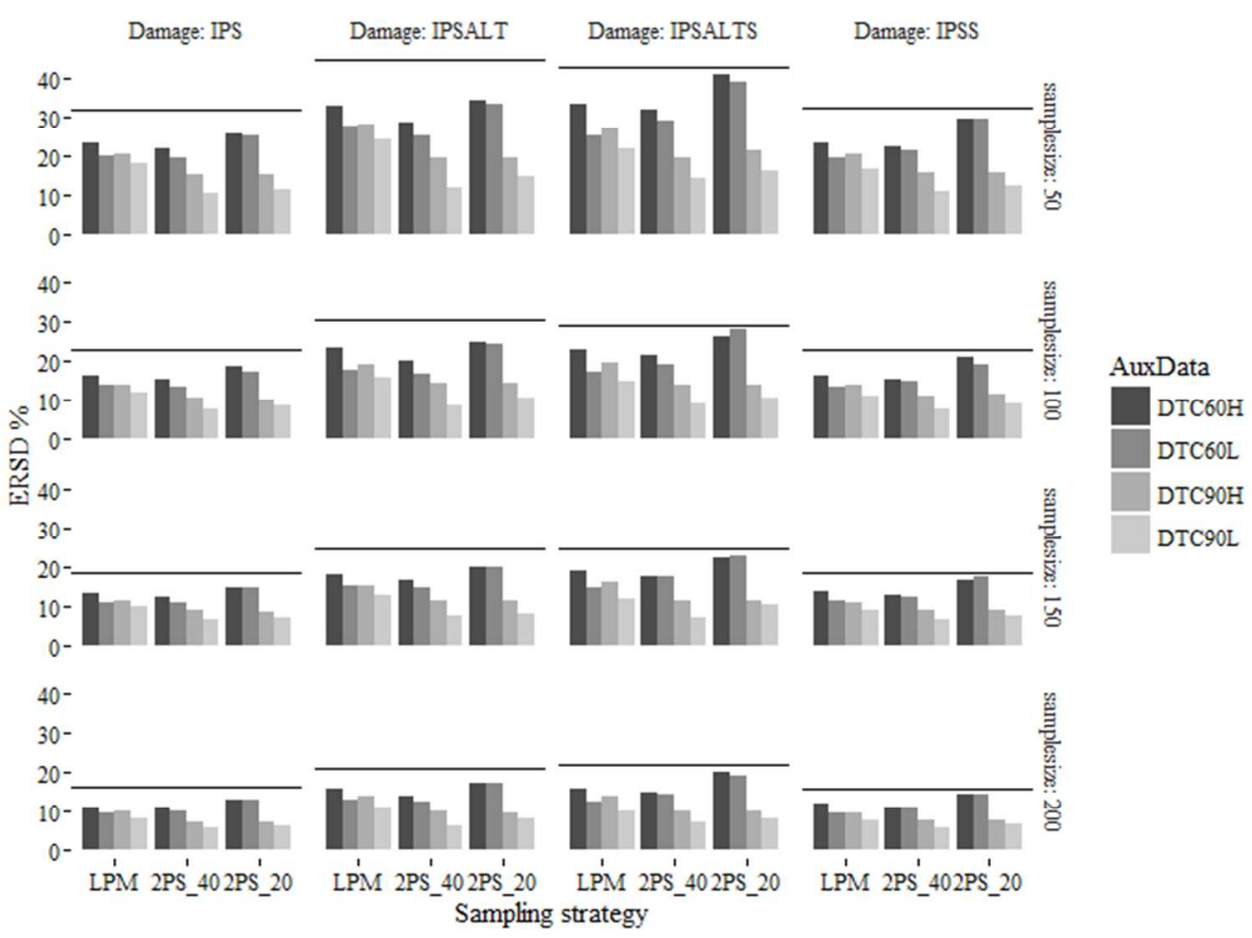

ERSD for $n \_1=10000$ and all combinations of damage, sample size, sampling strategy and auxiliary information used. ERSD for SI is included as a straight line.

Figure 2 


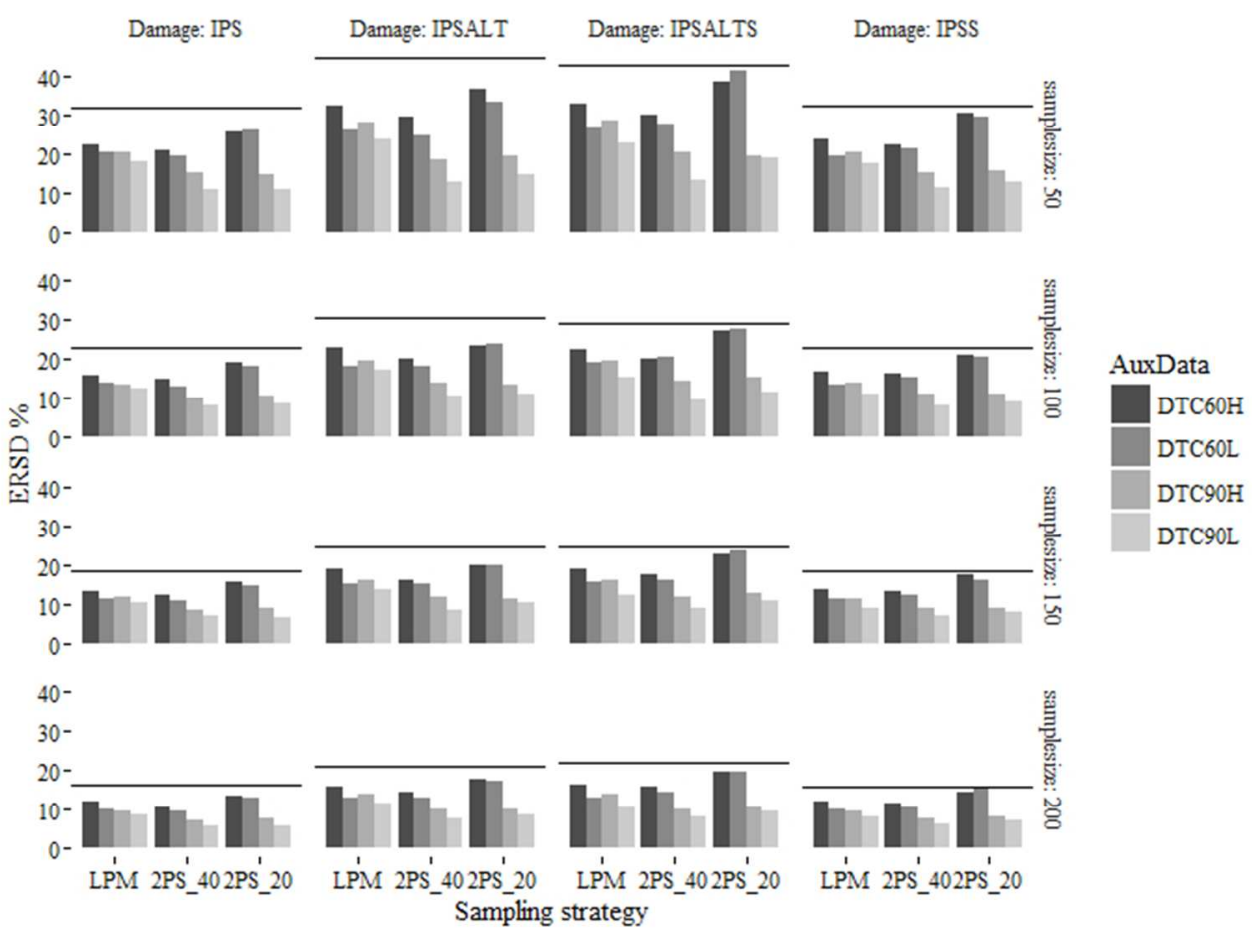

ERSD for $n \_1=3600$, and all combinations of damage, sample size, sampling strategy and auxiliary information used. ERSD from SI design in straight line across.

Figure 3 